\title{
Investigation of Friction Wear Process in the Presence of Exfoliated Graphite Nanoparticles using Correlative in situ Raman Micro Spectrometry and Post Mortem Raman, Analytical Scanning and Transmission Electron Microscopies
}

\author{
Audrey Molza ${ }^{1}$, Jean-Louis Mansot ${ }^{1,2}$, Yves Bercion ${ }^{2}$ and Farid Begarin ${ }^{2}$ \\ ${ }^{1}$ Groupe de Technologie des Surfaces et Interfaces (GTSI EA 2432), Université des Antilles et de la \\ Guyane, Faculté des Sciences Exactes et Naturelles, BP 250, 97157 Pointe à Pitre Cédex, France \\ 2. Centre Commun de Caractérisation des Matériaux des Antilles et de la Guyane $\left(\mathrm{C}^{3} \mathrm{MAG}\right)$, Université \\ des Antilles et de la Guyane, Faculté des Sciences Exactes et Naturelles, BP 250, 97157 Pointe à Pitre \\ Cédex, France
}

The lubrication strategies using solid nanoparticles as antiwear and friction reducer additives developped during the last decades are at the origin of extensive studies [1] in order to understand the action mechanisms of nanoparticles in the friction and wear reduction processes. As far as Raman micro spectrometry is a powerful technique for physical and chemical in situ analyses in a dynamic contact [2], the technique is used, in the present study, to characterize the pressure and lubricant film thickness distributions and to follow the structural evolution of nanoparticles of exfoliated graphite during their transit through the sliding interface. Complementary investigations on the tribofilm generated during friction tests are carried out using post mortem Raman, Analytical Scanning and Transmission Electron Microscopies in order to characterize the composition and the structure of this few nanometers thick surface film.

The lubricant used was a 5\% w/w dispersion of exfoliated graphite nanoparticles (TIMCAL) in pure 5P4E (ALDRICH). The measurements of the intensity and of the wavenumber shift of the Raman characteristic $1000 \mathrm{~cm}^{-1}$ band of 5P4E allowed us to quantitatively characterize the liquid thickness and pressure distributions in the sliding contact whereas, the graphite $1350 \mathrm{~cm}^{-1}$ (D band) and $1580 \mathrm{~cm}^{-1}(\mathrm{G}$ band) allowed us to follow the structural evolution $\left(\mathrm{I}_{\mathrm{D}} / \mathrm{I}_{\mathrm{G}}\right.$ intensity ratios related to carbon phases disorder[3]) induced by the transit of the nanoparticles through the sliding contact. In situ Raman experiments are carried out at $25^{\circ} \mathrm{C}$ on a rotating sphere (saphire) / plane (AISI 52100 steel) tribometer (built at lab.) coupled to the Raman micro-spectrometer (HORIBA HR 800) [2]. Post mortem analyses are carried out on tribofilms produced on reciprocating ball (52100 AISI steel) on plane (52100 AISI steel) tribometer. After rincing of the friction surfaces with pentane, the morphology, composition and structural disorder of the tribofilm are investigated using Raman and Analytical Scanning Electron Microscopy (Quanta 250, W cathode, Oxford X Max EDS detector). The tribofilm is then extracted using extrative polymer replica method and after deposition onto a holey carbon film and dissolution of the polymer replica, the sample is introduced in a Tecnai F20 Xtwin microscope running at $200 \mathrm{kV}$ fitted with EDAX SiLi EDS detector and a Quantum Gatan imaging filter for analytical and structural investigations.

Figure 1 summarizes some of the results obtained during the study. The film thickness and pressure distributions (Figure 1A and 1B) acquired using in situ Raman micro spectrometry show that the lubricant film thickness is constant $(0.5 \mu \mathrm{m})$ in the sliding contact and similar to the film thickness obtained with pure 5P4E (not shown). The pressure distribution presents the classical elliptic shape according to previous numerical simulations [2] the maximal recorded pressure being $0.9 \mathrm{GPa}$ at the 
center of the contact. As for the thickness, the pressure distribution does not present significant changes compared to pure 5P4E lubrication conditions (not shown).

The SEM secondary electrons image and Raman point spectra (Figure 1C and 1D) acquired on the wear scar at the end of the friction test reveals the presence of carbonaceous tribofilm on the friction surfaces. The $\mathrm{I}_{\mathrm{D}} / \mathrm{I}_{\mathrm{G}}$ intensity ratio profile across the wear scar allows us to evaluate the graphitic domain spatial expansion (Figure 1E), using the Tuinstra and Koenig formula [3]. It clearly reveals that disorder in the carbon phase increases from the outside toward the center of the wear scar. This disorder is induced by the high shear rate $\left(\approx 10^{5} \mathrm{~s}^{-1}\right)$ in the contact.

TEM Bright Field and Dark Field images (Figure 1F and 1G), electron diffraction pattern (Figure 1H) and EELS spectrum (Figure 1I) collected onto the tribofilm extracted from the wear scar confirm its less organized structure at the center of the wear scar. The film is mainly composed of graphitic nano domains which appeared as 100-200 nm extended areas. It is important to note that the graphitic nano domains are mainly oriented with the graphene layers parallel to the sliding surfaces as indicated by the (110) collected Dark Field image (Figure 1G). This specific orientation, resulting from the simultaneous presence of liquid and solid nano-particles in the sliding interface, is probably at the origin of the low friction coefficient obtained (0.08).
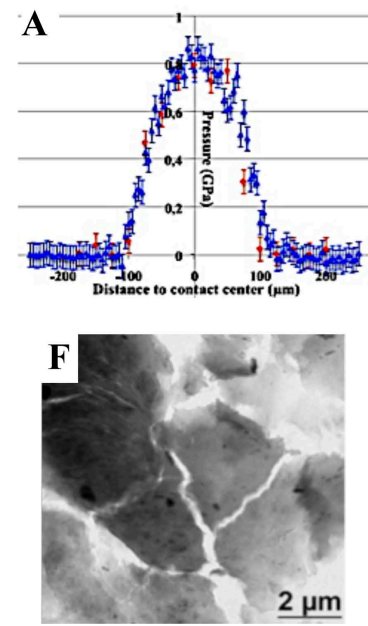
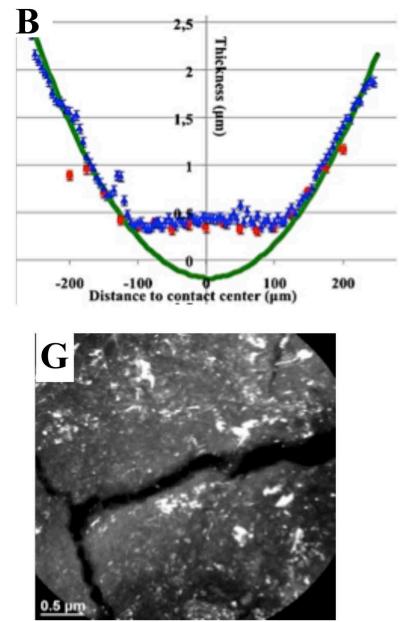
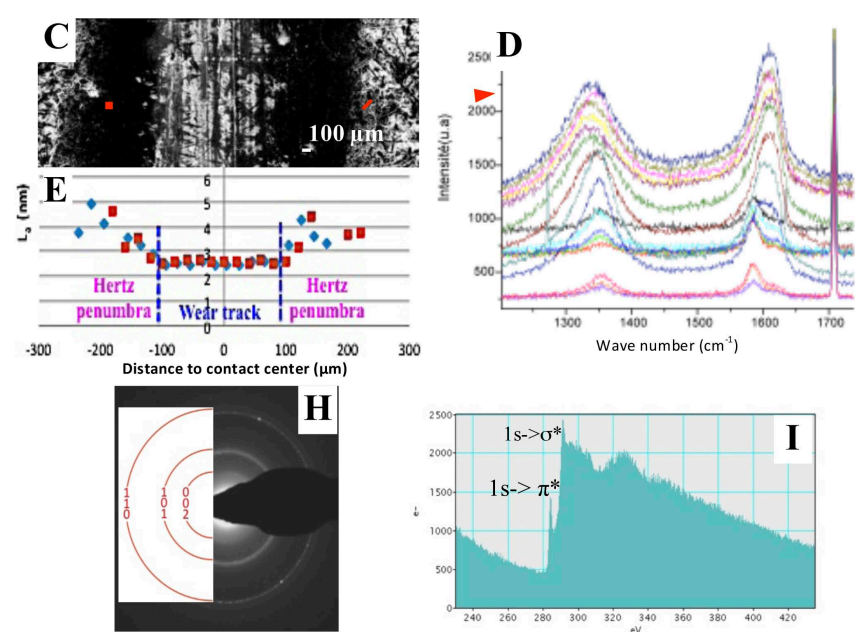

Figure 1. A, B) Pressure and film thickness distribution simultaneously measured using in situ Raman microscopy. C) SED image of the wear at the end of the test. D) Raman point spectra acquired across the wear scar (red dots in C). E) Evolution of the coherence length (La) as a function of the distance to the center of the wear scar. F, G, H) Bright and Dark (110) field images and corresponding electron diffraction pattern collected onto the tribofilm. I) EELS spectrum of the film.

\section{References :}

[1] J.M Martin, Ohmae O. "Nanolubricants" ed. Wiley, New York, (2008).

[2] D Himmel et al, "In situ Raman Microspectrometry of lubricated tribologic contacts. Part two:

Simultaneous measurements of Pressure, Lubricant Film thickness and Temperature distributions in a running EHD contact”,Tribo. Lett. (2011) 41(1), pp. 131-144.

[3] F Tuinstra, J.L Koenig, "Raman spectrum of graphite", J. Chem. Phys. (1970) 53(3), pp.1126-1130.

[4]The authors acknowledge the Ministère de la Recherche, the Conseil Régional de la Guadeloupe, the Conseil Régional de la Martinique, the Fonds Social Européen (FSE) and the Fonds Européens de

Développement Régional (FEDER) for their financial supports. 